Schneider, Ilona K.; Oberländer, Franz

\title{
Rostocker Modell - ein didaktischer Ansatz zur Planung und Gestaltung von Lerneinheiten im Sachunterricht
}

Tänzer, Sandra [Hrsg.]; Lauterbach, Roland [Hrsg.]: Sachunterricht begründet planen. Bedingungen, Entscheidungen, Modelle. Bad Heilbrunn : Verlag Julius Klinkhardt 2010, S. 203-223

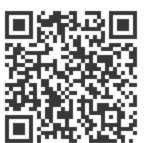

\section{Quellenangabe/ Reference:}

Schneider, llona K.; Oberländer, Franz: Rostocker Modell - ein didaktischer Ansatz zur Planung und Gestaltung von Lerneinheiten im Sachunterricht - In: Tänzer, Sandra [Hrsg.]; Lauterbach, Roland [Hrsg.]: Sachunterricht begründet planen. Bedingungen, Entscheidungen, Modelle. Bad Heilbrunn : Verlag Julius Klinkhardt 2010, S. 203-223 - URN: urn:nbn:de:0111-pedocs-209301 - DOI: 10.25656/01:20930

https://nbn-resolving.org/urn:nbn:de:0111-pedocs-209301

https://doi.org/10.25656/01:20930

in Kooperation mit / in cooperation with:

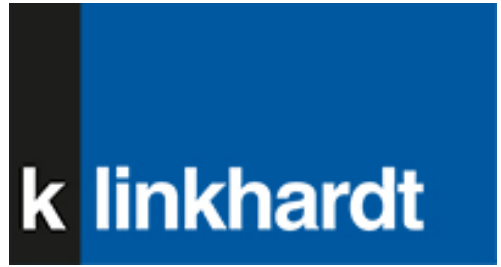

http://www.klinkhardt.de

\section{Nutzungsbedingungen}

Dieses Dokument steht unter folgender Creative Commons-Lizenz: http://creativecommons.org/licenses/by-nc-nd/4.0/deed.de - Sie dürfen das Werk bzw. den Inhalt unter folgenden Bedingungen vervielfältigen, verbreiten und öffentlich zugänglich machen: Sie müssen den Namen des Autors/Rechteinhabers in der von ihm festgelegten Weise nennen. Dieses Werk bzw. dieser Inhalt darf nicht für kommerzielle Zwecke verwendet werden und es darf nicht bearbeitet, abgewandelt oder in anderer Weise verändert werden.

Mit der Verwendung dieses Dokuments erkennen Sie die Nutzungsbedingungen an.

\section{Terms of use}

This document is published under following Creative Commons-License: http://creativecommons.org/licenses/by-nc-nd/4.0/deed.en - You may copy, distribute and transmit, adapt or exhibit the work in the public as long as you attribute the work in the manner specified by the author or licensor. You are not allowed to make commercial use of the work or its contents. You are not allowed to alter, transform, or change this work in any other way.

By using this particular document, you accept the above-stated conditions of use.

\section{Kontakt / Contact:}

peDOCS

DIPF | Leibniz-Institut für Bildungsforschung und Bildungsinformation

Informationszentrum (IZ) Bildung

E-Mail: pedocs@dipf.de

Internet: www.pedocs.de

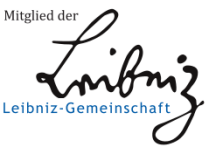




\title{
5.3 Rostocker Modell - ein didaktischer Ansatz zur Planung und Gestaltung von Lerneinheiten im Sachunterricht
}

\author{
von Ilona K. Schneider und Franz Oberländer
}

\subsubsection{Einleitung}

An der Universität Rostock im Bereich Grundschulpädagogik wurde für die Planung und Gestaltung von Lerneinheiten des Sachunterrichts ein didaktisches Modell entwickelt. Dieser Ansatz geht davon aus, dass Lernen ein langfristiger Prozess ist, der auf Instruktion, Selbsttätigkeit und Kooperation beruht, der die Schüler als Lernsubjekte tatsächlich ernst nimmt und der vor allem die Aneignung von vernetztem Konzeptwissen in den Mittelpunkt stellt. Das Rostocker Modell basiert auf verschiedenen lernpsychologischen Theorien und didaktischen Konzepten. ${ }^{1}$ Es wurde sowohl auf Themen des gesellschaftlich-sozialen Bereichs (Schneider 2007) als auch auf naturwissenschaftlich orientierte Lerneinheiten (Schneider \& Oberländer 2009; Schneider, Hruby \& Pentzien 2007) angewandt.

\subsubsection{Grundannahmen des Rostocker Modells}

Das Rostocker Modell ist eine didaktische Konzeption, die auf folgenden Grundannahmen beruht:

○ Lernen ist ein sozial-konstruktiver Aneignungsprozess.

- Lernprozesse haben einen langfristigen Charakter.

- Lernen ist auf die intrinsisch motivierte Eigenaktivität des Lerners und Instruktionen angewiesen.

- Nachhaltiges Lernen gründet sich auf Konzeptwissen.

- Schulisches Lernen ist eine Form der Enkulturation.

\footnotetext{
Es handelt sich um die Theorien von Vygotskij (2003), Bruner (1973), Poddjakow (1981) und Klafki (1957; 1985; 1992), um angloamerikanische didaktische Konzepte von Hodson (1998), Blythe (1998), Charles (2000) und Clarke (2001) und um die Ergebnisse unserer eigenen empirischen Untersuchungen: Toth u.a. (2007; 2008), Schneider \& Oberländer (2009), Revákné Markóczi u. a. (2008), Schneider u. a. (2008a; 2008b; 2009).
} 


\section{Lernen - ein sozial-konstruktiver Aneignungsprozess}

Lernen kann als eine mit Anstrengung verbundene bereichsspezifische Tätigkeit verstanden werden, bei der sich der Mensch die Welt gegenständlich, sozial und ideell relativ dauerhaft und anwendungsbezogen aneignet. Beim Lernen werden durch Konstruktion und Rekonstruktion mentale Modelle (Konzepte) ständig in Abhängigkeit der Erfahrungen aufgebaut, gefestigt, umgeformt, ergänzt usw. Diese Aneignungsprozesse werden in hohem Maße u. a. von weltanschaulichen, kulturellen, subkulturellen, emotionalen und situativen Faktoren mitbestimmt (Hodson 1998; Schneider 2003). Beim Lernen werden Informationen in den Assoziationsspeicher des Gehirns codiert, aus dem die Inhalte nach dem Ähnlichkeitsprinzip abrufbar sind. Die vorhandenen Informationsmuster bestimmen wesentlich mit, was wahrgenommen und wie es interpretiert wird. Auch wenn Informationen unvollständig sind, sucht das Gehirn nach passenden (,guten“) Interpretationsgestalten und fügt von sich aus „Fehlendes“ hinzu, ohne dass dieses der Person bewusst sein muss. Wahrnehmungen tragen Prozess- und Produktcharakter zugleich. Als Prozesse zeigen sie sich in einem erwartungsgesteuerten und damit stark vom aktuellen Wissen beeinflussten Suchen und Vergleichen; als Produkt manifestieren sie sich in Deutungen und Interpretationen, die wiederum als aktuelles Wissen die folgenden Wahrnehmungen vorstrukturieren (Singer 2003, 35 und 43).

Schüleradäquates didaktisches Handeln heißt, den einzelnen Schüler in die Lage zu versetzen, die neuen Anforderungen in sein Welt- und Selbstbild und in sein Handlungsrepertoire aufnehmen zu können. Ausgangspunkt sind hierbei seine aktuellen Erfahrungen (Wissen, Können), auf deren Grundlage er selbständig handeln kann. Doch diese Erfahrungen, von Vygotskij (2002) als Niveau der aktuellen Entwicklung bezeichnet, beschreiben den gegenwärtigen Entwicklungsstand des Kindes nur unzureichend. Um die Entwicklungschancen des schulischen Lernens zu nutzen, muss für jedes Kind auch die Zone der nächsten Entwicklung bestimmt werden. Damit bezeichnet Vygotskij die Differenz zwischen dem Niveau, auf dem das Kind die Aufgaben unter Anleitung lösen kann, und dem Niveau, auf dem es Aufgaben selbständig löst. Aufgaben und Übungen sind in der Zone der nächsten Entwicklung anzusiedeln. Hauptmerkmal dieses Lernstadiums ist die Zusammenarbeit. Die Zone der nächsten Entwicklung, also die Lernzone, ist auf sozialen Austausch und Input angewiesen: „... denn was das Kind heute in Zusammenarbeit zu leisten vermag, das wird es morgen selbständig auszuführen im Stande sein“ (348). Individuelle Selbstverwirklichung und sozialer Abgleich müssen bei der Gruppenarbeit also Hand in Hand gehen. Lernen ist immer ein zutiefst sozialer Vorgang. Die soziale Kooperation vollzieht sich in vielfältigen Formen. Sie umfasst praktische wie theoretische Lehrerinstruktionen, 
Partner- und Gruppenarbeit der Kinder untereinander, den Austausch mit Experten sowie die Nutzung von vielfältigen Medien.

\section{Lernen - ein langfristiger Prozess}

Kinder brauchen Zeit, um Ideen zu entwickeln. Sie brauchen Zeit zum Nachdenken, zum Formulieren ihrer Gedanken. Sie brauchen Zeit zur Wiederholung, zum Üben, zum Ausprobieren. Sie brauchen Zeit für ihre Fragen, für Gespräche. Sie brauchen auch noch in der Grundschule viel Zeit zum Spielen und zur Bewegung.

Kinder benötigen Impulse, um Aufmerksamkeit zu entfalten und auszudehnen. Sowohl Erwartungshaltungen als auch neue Reize und Reizankündigungen stimulieren eine selektive Aufmerksamkeit. Ist die Aufmerksamkeit erst einmal geweckt, brauchen Kinder Zeit, um ihre Aufmerksamkeit zu lenken, zu koordinieren und auszudehnen. Sie müssen Gelegenheiten haben, sich mit einem Objekt über eine genügend lange Zeit zu befassen, sich darauf zu konzentrieren, es mit anderen Objekten zu vergleichen und zwischen Objekten Beziehungen herzustellen. Die didaktische Antwort auf die Langfristigkeit von Lernprozessen realisiert sich in einer übergreifenden Planung, in der Planung von größeren, komplexen Lerneinheiten. Für diese Lerneinheiten werden Lernziele und Lernkriterien, die für alle Unterrichtsplanungen im Rahmen dieser Lerneinheit gültig sind, bestimmt, um eine den gesamten Lernprozess begleitende Orientierung und Motivation $\mathrm{zu}$ ermöglichen (Schneider, Oberländer 2008, 22).

\section{Intrinsisch motivierte Eigenaktivität der Kinder und Instruktionen}

Das Hauptanliegen besteht darin, Kinder tatsächlich als Subjekte ihrer Lernprozesse zu begreifen und ernst zu nehmen. Das bedeutet, sie bewusst und verantwortlich in die Lehr- und Lernprozesse einzubinden. Dazu müssen wir die Bedürfnisse der Kinder in der Schule kennen und bei der Gestaltung unserer Lehrtätigkeit berücksichtigen. Lernlust, Lernbereitschaft beginnen sich zu entwickeln, wenn das Kind

○ für sich einen Sinn, einen Nutzen in der Lernaufgabe erkennt,

- auf Erfolg und Bestätigung hoffen kann,

- seine Interessen angesprochen sieht,

- weiß, worum es geht, was es erreichen soll bzw. will,

- die Lernanforderungen herausfordernd und gleichzeitig erreichbar erlebt,

- seine Erwartung, dass sein Lernen erfolgreich sein wird, bestätigt sieht

(Hodson 1998; Charles 2000; Dean 2006).

Gerade die intrinsische Motivation ist ein ganz entscheidender Lernfaktor. In diesem Falle führt der Lernende die Lernhandlung um ihrer selbst wegen aus, weil er neugierig ist, weil ihn der Gegenstand interessiert, weil er sich ange- 
spornt fühlt usw. Gleichsam gilt es zu berücksichtigen, dass auch intrinsische Motivationen selbst sehr unterschiedlich angelegt sein können. Jeder Lerner, also auch jedes Kind, bildet sein ganz spezifisches motivationales Muster aus. Die Kenntnis, welchen Motivtyp ein Kind ausgebildet hat, ist hilfreich zur Bestimmung seiner bevorzugten Art des Lernens (Hofstein \& Kempa 1985; Kempa \& Diaz 1990).

Tab. 1: Durch Motivationsmuster beeinflusstes Lernen

\begin{tabular}{|l|l|}
\hline Motivtyp & Bevorzugtes Lernen \\
\hline Leistungsbedürfnis & Eine auf Wettbewerb ausgerichtete Lernumgebung \\
\hline Neugierbefriedigung & $\begin{array}{l}\text { Selbstgesteuertes und auf Problemlösung ausgerichtetes } \\
\text { Lernen }\end{array}$ \\
\hline Pflichterfüllung & Klar definierte Ziele und eindeutige Instruktionen \\
\hline $\begin{array}{l}\text { Bedürfnis nach sozialem } \\
\text { Anschluss }\end{array}$ & Eine auf Kooperation ausgerichtete Lernumgebung \\
\hline
\end{tabular}

Aufgrund der unterschiedlichen Motivationen für schulisches Lernen wird die Lernumgebung variiert und flexibel gestaltet, um den Bedürfnissen eines jeden Kindes gerecht zu werden (Charles 2000). Die kognitive Entwicklung, die bei Lernprozessen zum Ausdruck kommt, wird durch unterschiedliche emotionale Befindlichkeiten begleitet. West und Pine (1983) nennen vier mögliche Gefühlsaspekte:

○ Kinder empfinden durch wachsende Kompetenz ein Gefühl der Stärke.

- Kinder nehmen wahr, wie sich komplexe Sachverhalte vereinfachen, wie sie überschaubarer und durchschaubarer werden.

- Kinder entwickeln ästhetische Empfindungen für Schönheit, Harmonie und Stimmigkeit des Lerngegenstandes.

- Kinder spüren ein Wohlbefinden und fühlen sich in ihrer persönlichen Integrität gestärkt.

Das menschliche Gehirn ist so ausgelegt, dass es viele Informationen von anderen Menschen aufnehmen und verarbeiten kann. Gerade Kinder sind darauf angewiesen, insbesondere von Erwachsenen zu lernen, da sie die Menge der für sie notwendigen Informationen unmöglich alle selbst beschaffen können (Gopnik 2000). Das gilt natürlich auch für schulisches Lernen. Die geistigen Strukturierungsleistungen der Kinder werden durch zusammenfassende, erklärende oder informierende Instruktionen des Lehrers nachhaltig unterstützt. Außerdem sind die enge Verbindung von Kognition und Emotio- 
nen (Csikszentmihalyi 1985) beim Lernen zu berücksichtigen. Deshalb achtet der Lehrer bei der Organisation des Lernprozesses darauf, dass

- die Kinder ihre Aufmerksamkeit auf ein beschränktes, fest umrissenes Handlungsfeld richten ( $\rightarrow$ Kenntnis der Lernziele),

$\circ \quad$ es eine Vielfalt von Handlungsmöglichkeiten im Rahmen des fest umrissenen Handlungsfeldes für die Kinder gibt ( $\rightarrow$ Möglichkeit von Selbsttätigkeit und Kooperation),

$\circ \quad$ die Handlungsanforderungen zusammenhängend und eindeutig sind und mit klaren, wiederum eindeutigen Rückmeldungen einhergehen $(\rightarrow$ Kenntnis der Lernkriterien),

○ er jedem Kind die Überzeugung vermittelt, die Lernaufgabe bewältigen zu können, wenn es sich anstrengt, bei Problemen um Hilfe bittet und mit anderen Kindern zusammen arbeitet ( $\rightarrow$ Stärkung des Selbstwertgefühls),

○ er alle möglichen Störquellen ausschließt $(\rightarrow$ selektive und polarisierte Aufmerksamkeit).

\section{Nachhaltiges Lernen und Konzeptwissen}

Jeder Mensch besitzt aufgrund seiner tagtäglichen Auseinandersetzungen mit der ihn umgebenden Lebenswelt ein umfangreiches Konzeptwissen, so genannte Alltagskonzepte, die sich als Denk- und Handlungsorientierungen im Alltag bewähren. Wissenschaften bilden innerhalb der Alltagswelt relativ eigenständige, kulturell geprägte und historisch gewachsene Bereiche (Berger \& Luckmann 1991), die sich u. a. durch spezifische Handlungen, Sprachmuster und Begriffe, durch Verallgemeinerungen, Idealisierungen und Symbolisierungen auszeichnen (Singer 2003). Zwischen Alltagskonzepten und den wissenschaftlichen Konzepten gibt es, trotz grundsätzlicher Unterschiede, eine starke wechselseitige Beeinflussung. Alltagskonzepte bilden die Grundlage, auf der sich wissenschaftliche Vorstellungen schrittweise ausbilden. Sie entwickeln sich vom Besonderen zum Allgemeinen. Wissenschaftliche Konzepte dringen nach und nach in die Alltagskonzepte ein. Sie erklären vom Allgemeinen ausgehend das Besondere. 
Tab. 2: Merkmale von Alltagskonzepten und wissenschaftlichen Konzepten (Vygotskij, 2002)

\begin{tabular}{|l|l|}
\hline Alltagskonzepte & Wissenschaftliche Konzepte \\
\hline - spontane Konzepte & - sind allgemein, abstrakt und idealisiert \\
- hohe persönliche Bedeutsamkeit & - mit anderen allgemeinen Konzepten \\
- meist lokal gebunden und isoliert & verbunden \\
von anderen Konzepten & $\begin{array}{l}\text { - werden vor allem deduktiv angeeignet } \\
\text { - } \begin{array}{l}\text { - ihr Verden vorwiegend induktiv ange- } \\
\text { eignet }\end{array}\end{array}$ \\
\hline
\end{tabular}

Nachhaltiges Lernen zeigt sich im Verstehen. Einen Inhalt zu verstehen bedeutet, in der Lage zu sein, über ein Thema auf die unterschiedlichste Weise nachzudenken, es zu erklären, seine Bedeutung herauszufinden, mit Beispielen zu illustrieren und auf andere Sachverhalte, insbesondere auch auf Alltagssituationen, sinnvoll zu übertragen und gegebenenfalls zu variieren. Verstehen ist nicht gleich Wissen, aber Wissen liegt dem Verstehen zugrunde (Blythe 1998). Verstehen zeigt sich in einem gewissen Kompetenzgrad der Anwendung allgemeiner, übergreifender Konzepte. Damit sind Denk-, Handlungs- und Erklärungsmuster gemeint, die helfen, in komplexen Situationen sinnvoll handeln zu können. Je allgemeiner ein Konzept ist, desto größer ist sein Anwendungsbereich, desto intensiver ist seine Nachhaltigkeit.

Die Frage ist, wie kann Unterricht ein nachhaltiges Lernen bewirken? Generelle Strategien zur Ausbildung eines derartigen Konzeptwissens sind z.B. eine systematische Analogiebildung, das Finden von möglichst vielen verschiedenen Beispielen, das Erkennen von Gemeinsamkeiten und Unterschieden, die Untersuchung von Extremfällen, Veranschaulichungen und Modellierungen bei gleichzeitiger Begriffsbildung, Präsentationen in unterschiedlichen Systemen, die Durchführung von Gedankenexperimenten und nicht zuletzt das Nachdenken über Dinge, Ereignisse, Geschehnisse und das Lernen selbst. Dazu ist ein exemplarisches Thema (Klafki 1957) vertieft und mit voller Aufmerksamkeit und entsprechender Zeitinvestition zu behandeln. Ausgangspunkte sind nach einer Sensibilisierungsphase, in der auch die vorhandenen Kenntnisse der Kinder deutlich werden, die Klärung der Lernbedeutung des Themas, die Bestimmung der Lernziele und die Festlegung von Lernkriterien für die Kinder. Zu Beginn der Erkundungsphase schafft der Lehrer eine allgemeine kognitive Orientierungsgrundlage: Er gibt Muster, Beispiele vor, begründet diese Orientierungen und hält erklärende Informationen bereit. Die Kinder erhalten Gelegenheit, praktisch-gegenständliche Handlungen in sozialen Lernarrangements durchzuführen. Der Lehrer moti- 
viert zum genauen Arbeiten, achtet auf Vollständigkeit der Teilhandlungen, gibt fortlaufende Rückmeldungen und schafft Möglichkeiten zur Qualifizierung der Lernergebnisse. In der zusammenfassenden Präsentationsphase, die einen Kontrollcharakter besitzt, fordert der Lehrer die Kinder auf, Sachverhalte und Erkenntnisse in unterschiedlichen Präsentationsformen, wie Modellierung, Grafiken, Übersichten, Schemata, darzustellen und ausgewählte Handlungen mündlich und/oder schriftlich vollständig zu beschreiben. Zum Abschluss der Lerneinheit denken die Kinder über ihr Lernen und ihren Lernfortschritt sowie über ihre Gefühle, die sie beim Lernen hatten, in einer Reflexionsphase nach.

\section{Schulisches Lernen - eine Form der Enkulturation}

Naturwissenschaftliches Lernen wird vorrangig durch einen Wechsel der Konzepte vom Alltagsverstehen zu einem wissenschaftlichen Verständnis erklärt (Carey 1985; Duit 1997; Hodson 1998). Aber auch viele Bereiche der sozialen Welt unterscheiden sich in ihren Handlungs- und Verhaltensmustern sowie in ihren Begriffs- und Symbolsystemen von der Alltagswelt (Schneider 2007). Beim schulischen Lernen werden Kinder schrittweise in für sie neue Handlungsdomänen einschließlich deren Begriffssysteme eingeführt. Aus dieser Perspektive kann schulisches Lernen als ein Enkulturationsprozess aufgefasst werden. Dieser Enkulturationsprozess ist sowohl auf eine gestaltete Praxis, die sich in einer aktiven Partizipation der Kinder und der Berücksichtigung ihrer Interessen zeigt, als auch auf Anleitung und Instruktion angewiesen. Das bedeutet, dass schulisches Lernen immer auch deduktive Lehrpassagen beinhalten muss. Ohne die richtigen Begriffe können Kinder nichts beschreiben, erklären und verstehen. Begriffe helfen sich auszudrücken und sich adäquat zu verständigen. Zudem, es gibt keine „reinen“ Beobachtungen. Beobachtungen werden immer auf der Basis des aktuellen Wissens, von Glauben, Erwartungen und Erfahrungen interpretiert, d.h. sie sind immer theorieabhängig - auch wenn diese Theorien Alltagstheorien sind. Beobachtungen brauchen einen Anreiz, einen Fokus der Aufmerksamkeit, einen Zweck. Gleichsam werden sie von der Sprache, den zur Verfügung stehenden und verwendeten Begriffen geprägt. Kinder erlangen neue Konzepte nicht durch Beobachtungen, sondern durch die Anwendung von Konzepten, die ihren Beobachtungen Sinn verleihen (Hodson 1998; Krnel 2005).

Deshalb kann schulisches Lernen auch als Enkulturation, als ein Hineinwachsen in neue Kulturen, verstanden werden, die sich durch angeleitete Teilnahme und gestaltete Praxis, die spezielle Inhalte der menschlichen Lebenswelt aufgreift, welche die Interessen der Akteure ansprechen und für weiterführendes Lernen bedeutsam sind, vollzieht. Dem Lehrer kommt hier- 
bei eine ganz entscheidende Funktion zu. Er muss im wahrsten Sinne des Wortes bereits „enkulturiert“ sein. Das bedeutet, er beherrscht die entsprechenden Inhalte und Methoden als Experte und ist in der Lage, diese zu vermitteln. Zunächst stellt der Lehrer fest, welche Vorstellungen die Kinder über das anzueignende Phänomen bereits haben. Gemeinsam werden Lernbedeutung, Lernziele und Lernkriterien der Lerneinheit herausgearbeitet und verdeutlicht.

Die Kinder werden zu einem neuen bzw. höheren konzeptionellen Verständnis sozialer Situationen bzw. von Gesetzmäßigkeiten der Natur durch soziale Interaktionen und damit einhergehende Kommunikation geführt. Dieser Ansatz kann als reziprokes Lehren bzw. reziprokes Lernen bezeichnet werden (Gardner 1994, 274). Er realisiert sich in der anfänglichen Führungsrolle des Lehrers, einer passenden Unterstützung für jedes Kind und in dessen zunehmender selbstständiger Partizipation am Lernprozess. Im Verlauf des Lernprozesses arbeiten die Kinder mit zunehmendem Verständnis immer autonomer (Hodson 1998). Die soziale Zusammenarbeit zwischen dem wissenden Lehrer und den lernenden Kindern bzw. zwischen Kindern, die den Lernstoff schon verinnerlicht haben und solchen, die sich noch im Lernstadium befinden, ist das entscheidende Instrument bei der zunehmend wissenschaftlichen Enkulturation der Kinder.

Die Gestaltung von langfristigem, nachhaltigem, auf Erwerb von Konzeptwissen orientiertem Lernen ist Ziel der folgenden didaktischen Konzeption.

\subsubsection{Rostocker Modell - didaktische Handlungsprinzipien}

Die Planung und Gestaltung eines Unterrichts nach dem Rostocker Modell zeichnet sich durch folgende didaktische Handlungsprinzipien aus:

- Diskussion der Bedeutung des Lerninhalts

- Ermittlung einer Leitidee

○ Formulierung gemeinsamer Lernziele

- Erarbeitung von konkreten Lernkriterien

$\circ \quad$ Ermittlung der Lernvoraussetzungen der Kinder

- Verbindung von Selbsttätigkeit und Instruktion

○ Entwicklung einer kommunikativen Gesprächs- und Fragekultur

- Lernprozessbegleitende Verbindung von Selbstbewertung und Rückmeldung

- Stimulierung der Selbstreflexion über das eigene Lernen

○ Stärkung der Selbstachtung eines jeden Kindes.

\section{Diskussion der Bedeutung des Lerninhalts}

Eine didaktische Grundidee dieses Modells besteht darin, Lerner von Anfang an aktiv in eine für sie durchschaubare und abrechenbare Organisation der Lernprozesse einzubeziehen. $\mathrm{Zu}$ Beginn einer neuen Lerneinheit diskutiert 
die Lehrerin gemeinsam mit den Lernern die Bedeutung der Lernthematik: „Warum wollen wir das lernen?"; „Wozu ist das wichtig?“. Kinder (und nicht nur sie) fragen oft, welchen Sinn bzw. Nutzen hat das zu Lernende für mich (Schneider 2003, 22). Sie machen eine Art „Aufwand-Nutzen-Rechnung“ und nehmen im Wesentlichen nur das auf, was sie für sinnvoll halten. Deshalb sind Themen auszuwählen bzw. so darzustellen, dass die Kinder in ihrem gegenwärtigen Leben deren Bedeutung erkennen können. Für jüngere Kinder ist es wichtig, natürliche Phänomene aus ihrer unmittelbaren Umgebung zu untersuchen. Sie brauchen kognitive Herausforderungen, die sie bewältigen können. Dazu gehören auch „passende Untersuchungen“, die ein klares Ziel haben und „funktionieren“ und insgesamt die Entwicklung geistiger Konzepte unterstützen.

Die Kinder müssen wissen, warum sie etwas lernen. Die Bedeutung des Lerninhalts ist im Rahmen eines Schüler-Schüler-Gespräches (Schneider 2000) zu diskutieren. Unterstützend wirkt die Einordnung des konkreten Lerninhalts in einen übergeordneten Zusammenhang. Diese Vorgehensweise kommt auch der Arbeit des menschlichen Gehirns entgegen. Das Gehirn agiert in einer ständigen Erwartungshaltung und gleicht die eingehenden Informationen damit ab. Wenn also durch „Vorreize“ die Aufmerksamkeit erhöht wird, können Lernprozesse intensiviert werden (Spitzer 2004).

Das Lernen der Kinder wird positiv beeinflusst, wenn die Lernbedeutung in einer für die Kinder verständlichen Sprache formuliert wird und während des gesamten Lernprozesses ihnen stets sichtbar vor Augen, z.B. an einer Tafel oder auf einem Poster, steht (Clarke 2001). Während des Lernprozesses ist immer wieder auf die Bedeutung des zu Lernenden Bezug zu nehmen.

\section{Ermittlung einer Leitidee}

Wie wir bereits dargelegt haben, ist es sehr wichtig, sich allgemeine, übergreifende Konzepte anzueignen, um in komplexen Alltagssituationen adäquat handeln zu können. In diesen Konzepten kommt das Elementare eines Lernthemas zum Ausdruck. Das Elementare verweist auf ein allgemeines Prinzip, das im exemplarisch behandelten Inhalt enthalten ist (Klafki 1957). Dieses Elementare kann sich als Leitidee eines Lerninhalts präsentieren, die die Eigenschaften hat, weiterführende, übergreifende, grundlegende Gedanken zu produzieren. Leitideen können z.B. in Form offener Fragen oder als Sprichwort bzw. Lebensweisheit formuliert werden. Sie dürfen didaktisch nicht streng verplant werden.

Eine Leitidee kann man sich auch als ein durchgängiges Motiv einer Lerneinheit (ähnlich einem musikalischen Motiv eines Musikstückes) vorstellen, das immer wieder auf den Grundgedanken verweist und ihn in immer neuen Perspektiven inhaltlich öffnet. Die inhaltliche Positionierung muss jeder 
selbst vornehmen. Eine derartige Idee präsentiert sich in Gegensätzen, Widersprüchen, sie lässt viele Perspektiven zu, die nicht auf ,richtig“ oder „falsch“ aus sind, die es den Schülern erlauben, ihre eigene Meinung zu formulieren. Deshalb sind Leitideen eng mit der Ausbildung eines Konzept- und Strategiewissens verbunden. Sie unterstützen durch ihren integrativen und vernetzenden Charakter das Erkennen von Zusammenhängen und den Wissenstransfer in andere Bereiche und damit auch das Verstehen (Blythe 1998).

Für die Identifikation einer Leitidee ist genügend Zeit einzuplanen. Sie kann über die Entwicklung eines Gedankennetzes (concept mapping) zu einem Lerninhalt gemeinsam mit den Kindern bestimmt werden. Im Rahmen eines Brainstormings werden alle Gedanken und Überlegungen zu einem Lerninhalt in Form eines Netzes aufgezeichnet. Die dabei entstehenden „Knotenpunkte" verweisen auf mögliche Leitideen. Damit ist noch einmal gesagt, dass zu einem Lerninhalt durchaus mehrere Leitideen auszumachen sind und man sich, bezogen auf die konkrete Lernsituation, für eine entscheiden muss (Blythe 1998).

\section{Formulierung gemeinsamer Lernziele}

Die Aufmerksamkeit für ein Lernthema wird in einem erheblichen Maße über Erwartungshaltungen stimuliert. Doch nicht nur das. Lernen ist überhaupt ein stark erwartungsgesteuerter und damit stark vom „Vorwissen“ beeinflusster Such- und Interpretationsprozess. Um an Erwartungshaltungen anzuknüpfen, sie zu stimulieren und über den gesamten Lernprozess aufrecht zu erhalten, greift eine an den Anfang einer Lerneinheit gestellte Motivationsphase zu kurz. Ein instrumenteller Einsatz der Lernziele über die Lehrerperspektive hinaus erweist sich hier als bedeutend wirkungsvoller.

Traditionell bestimmt die Lehrerin in der Vorbereitung einer neuen Lerneinheit die Lernziele bezogen auf anzueignendes Wissen, auszubildende Fähigkeiten und anzubahnende Einstellungen (Lehrerperspektive). Neu ist, dass sie diese Ziele in zwei Formen formuliert: einmal als offene Frage, die das Lernthema fokussiert (Was gilt es zu klären: Inwiefern ist das Ereignis $\mathrm{X}$ dem Ereignis $\mathrm{Y}$ ähnlich bzw. worin unterscheiden sich beide voneinander?) und einmal als Feststellung, die präzise das anzueignende Wissen beschreibt (Die Schüler erkennen, lernen, wissen etc.).

Um die Erwartungen der Kinder als Lerninstrumentarium zu nutzen, ist diese Art der Lernzielbestimmung für einen optimalen Lehr- und Lernprozess noch nicht ausreichend. Zudem gehen wir davon aus, dass Lernprozesse umso erfolgreicher verlaufen, wenn Kinder

- Klarheit über Lernaufgaben und Lernziele haben sowie

○ selbst Entscheidungen treffen und ihren Lernprozess kontrollieren können. 
Kinder brauchen von Anfang an eine fortlaufende (also immer sichtbare) Orientierung über die Zielstellungen der Lerneinheit. Klare Ziele sind Andockstationen für Erwartungen. Deshalb sind die Lernziele einer Lerneinheit nicht nur aus der Lehrerperspektive, sondern immer auch aus der Schülerperspektive zu bestimmen. Nachdem die Lehrerin die Bedeutung des neuen Thematik mit den Kinder geklärt hat, bespricht sie mit ihnen auch die angestrebten Lernziele: „Was sollen wir wissen und können?“ Die Lernziele werden in einer für die Kinder verständlichen Sprache und vor allem sehr konkret formuliert (Kinderperspektive) und ebenfalls für alle sichtbar im Klassenzimmer ausgehangen, damit sie sich daran fortlaufend orientieren können. Während des Lernprozesses wird immer wieder darauf Bezug genommen.

\section{Erarbeitung von konkreten Lernkriterien}

Überprüfungen und Rückmeldungen brauchen für ihren lernfördernden Einsatz orientierende Kriterien. Den Ausgangspunkt zur Bestimmung von Lernkriterien bilden die Lernziele. Die Lehrerin ist aufgefordert, bereits zu Beginn der Lerneinheit, die Kriterien zu bestimmen, an denen sie die Leistung ihrer Schüler messen wird. „Was muss ein Schüler im Niveau X wissen bzw. können?" Eine präzise Kennzeichnung der Leistungsniveaus erlaubt die Bestimmung des aktuellen Entwicklungsniveaus eines jeden Schülers und die Bestimmung der Zone seiner nächsten Entwicklung. So kann die Lehrerin eine zielgerichtete Unterstützung durch Instruktionen und passende Lernaufgaben organisieren. Bekanntermaßen verbinden sich Erkenntnisprozesse und die damit einhergehenden Emotionen in einer produktiven Weise, wenn die Lehrerin bei der Organisation des Lernprozesses darauf achtet, dass die Kinder ihre Aufmerksamkeit auf ein beschränktes, fest umrissenes Handlungsfeld richten und die Handlungsanforderungen zusammenhängend und eindeutig sind und mit klaren, wiederum eindeutigen Rückmeldungen einhergehen (Csikszentmihalyi 1985).

Ähnlich wie die Lernziele werden auch die Lernkriterien nicht nur aus der Lehrerperspektive (Analyse- und Bewertungsinstrumentarium), sondern auch aus der Schülerperspektive am Anfang einer neuen Lerneinheit formuliert. Während des gesamten Lernprozesses sollen den Schülern neben den Zielen auch die Kriterien bekannt sein, an denen sie und ihre Lehrerin erkennen können, ob und in welchem Maße die Lernziele erreicht worden sind. Es ist sehr zu empfehlen, dass die Lernkriterien für die Schüler (Schülerperspektive) gemeinsam von Lehrerin und Schülern erarbeitet und in der Sprache der Kinder festlegt werden: „Woran sehe ich, was ich bereits kann?“, „Welche Aufgaben kann ich noch nicht oder nur zum Teil lösen?“ „Wo brauche ich noch Hilfe?“. Lernkriterien aus der Schülerperspektive eröffnen den Schülern die Möglichkeit, über ihren Lernfortschritt kritisch nachzudenken und sich 
selbst einzuschätzen. Sie werden im Klassenraum veröffentlicht oder jedem Schüler in Listenform ausgehändigt, so dass sie immer präsent sind.

\section{Ermittlung der Lernvoraussetzungen der Kinder}

Laut Heran-Dörr verfügen viele Lehrerinnen und Lehrer nicht über angemessene Kenntnisse darüber, dass sich kindliche Vorstellungen durch einen Konzeptcharakter, relative Stabilität und potentielle Fehlerhaftigkeit auszeichnen (2006, 169f.). Wenn Lehrer Alltagserfahrungen der Kinder zu Beginn des Unterrichts aktivieren, dann vor allem als Motivationselement in einem Unterrichtsgespräch. Aber erst die diskursive Auseinandersetzung mit den eigenen Erfahrungen macht diese zu einem wichtigen Lernfaktor. Es ist eine entscheidende Frage, wie diese Vorstellungen didaktisch in Unterrichtssituationen genutzt werden können.

Ausgangspunkt kann ein Brainstorming in einer Assoziationsphase sein. Die Gedanken und Ideen der Kinder, die von ihnen verwendeten Begriffe, werden zunächst gesammelt. In einem zweiten Schritt sollen die Kinder die Begriffe in eine sinnvolle Zuordnung bringen. Hier bietet sich der Einsatz des Concept Mapping als ein Instrument zur bereichsspezifischen Erfassung des deklarativen Wissens eines Menschen an. Es ermöglicht die Feststellung des aktuellen Wissensstandes sowie dessen Veränderung im Verlauf einer bestimmten Zeiteinheit (Novak 1998). Der Gebrauch der Begriffe und ihre Zuordnung untereinander lassen auf den Grad des Verstehens schließen. Die Frage ist also, welche Begriffe kennen die Kinder und wie können sie diese prädikativ untereinander verbinden. Neben dem Concept Mapping können SchülerSchüler-Gespräche zur Ermittlung der Lernvoraussetzungen eingesetzt werden. Diese Methode eignet sich zur diskursiven Auseinandersetzung mit dem Problem und unterstützt seine reflexive Durchdringung (Schneider 2000, 68ff.). Schülervorstellungen lassen sich auch durch eine Kombination von bildlicher Darstellung (Schülerzeichnung) und einer Bildinterpretation erfassen. Die Wahl und der Gebrauch (ebenso der Nicht-Gebrauch) von Begriffen und die verwendeten Zuordnungen lassen Rückschlüsse auf das explizite Wissen zu (Toth 2007).

\section{Verbindung von Selbsttätigkeit und Instruktion}

Lernen ist ein komplexer Prozess, dem unterschiedliche Mechanismen zugrunde liegen. In jedem Falle ist Lernen immer an die Selbsttätigkeit des Lerners gebunden. Diese Selbsttätigkeit vollzieht sich sowohl im gegenständlichen als auch im geistigen Handeln. Deshalb ist es notwendig, den Kindern ausreichend Raum zum praktisch-gegenständlichen Handeln zu geben und ihnen gleichzeitig viele Gelegenheiten einzuräumen, über ihr praktisch-gegenständliches Handeln nachzudenken. 
Gleichzeitig ist aber das menschliche Gehirn darauf programmiert, möglichst viele Informationen von anderen Menschen aufzunehmen und zu verarbeiten. Kinder sind darauf angewiesen, dass Erwachsene Informationen an sie weitergeben, die frühere Generationen angesammelt haben. Von Erwachsenen erhalten sie, wie bereits ausgeführt, zahlreichere und bessere Informationen als sie sich selbst beschaffen können (Gopnik 2000). Die geistigen Strukturierungsleistungen werden somit nachhaltig unterstützt, wenn der Lehrer an bestimmten Stellen des Lernprozesses den Kindern zusammenfassende, erklärende oder informierende Instruktionen gibt. Bei Lernprozessen, die in eine andere Kultur, in eine spezifische Wirklichkeit, wie z.B. die Wissenschaften, einführen und deshalb vor allem deduktiv zu organisieren sind, kommt der Weitergabe von Informationen eine besondere Bedeutung zu. Instruierendes und selbsttätiges Lernen sind keine konkurrierenden Verfahren, sondern stehen in einer dialektisch-komplementären Beziehung zueinander. Beide Verfahren initiieren und unterstützen mentale Konstruktionen und Rekonstruktionen.

\section{Entwicklung einer kommunikativen Gesprächs- und Fragekultur}

Mit der Entwicklung einer kommunikativen Gesprächskultur wird den Kindern eine Kultur der Nachdenklichkeit nahe gebracht und sie werden zu einer diskursiven Auseinandersetzung mit den Lerninhalten angeregt. Insbesondere geht es um die Entwicklung einer schülerdominierten Kommunikationsform in Lernsituationen, um thematische Schüler-Schüler-Gespräche, die sich u.a. durch folgende Merkmale auszeichnen: Die Gesprächsteilnehmer sind gleichberechtigt, jede Meinung bereichert die Diskussion, gedankliche Freiheit ist gewährleistet und Gedankenexperimente sind erwünscht.

Die Kinder lernen, dass ein zeitweiliges Nichtverstehen und sich daraus ergebende Fragen natürliche und sehr wichtige Elemente eines Lernprozesses sind. Schwierigkeiten zu haben, sich anstrengen zu müssen, bedeutet, dass sich neues Lernen ankündigt. An dieser Stelle ist es wichtig, dass Kinder in der Lage sind, ihre Probleme zu artikulieren und um Hilfe zu bitten. Wir gehen davon aus, dass Fragen und Vermutungen der Kinder sowie deren Fähigkeit, bestimmte Aufgaben unter Anleitung lösen zu können, eine sensibilisierte Lernbereitschaft signalisieren. Fragen von Kindern sind nie zufällig, auch wenn sie Erwachsenen als zufällig erscheinen. Insbesondere die Reformpädagogik sah in Kinderfragen eine sprudelnde Lernquelle. „In dem Augenblick, wo das Kind fragt, interessiert sich das Kind unzweifelhaft für die Sache, denn dann ist sein Geist darauf gespitzt, die Erkenntnis aufzunehmen" (Otto 1965, 11).

Nach dem Rostocker Modell steht am Anfang jeder komplexen Lerneinheit die diskursive Durchdringung der Problematik, die u. a. die Bedeutung der 
Lerninhalte thematisiert und, wie oben dargelegt, zur Formulierung von Lernzielen und Lernkriterien aus der Schülerperspektive führt. Während des Lernprozesses werden immer wieder ein reflexives Nachdenken über den Lernfortschritt, über Lernprobleme und damit verbundene subjektive Befindlichkeiten angeregt. Jede Lerneinheit ist mit einer intensiven Reflexionsphase abzuschließen. Deshalb ist die Entwicklung einer kommunikativen Gesprächs- und Fragekultur von Beginn der ersten Klasse an notwendig, um die Kinder zu befähigen, einen diskursiven Gedankenaustausch führen und sich aktiv in den Lernprozess einbringen zu können.

\section{Lernprozessbegleitende Selbstbewertung und Rückmeldung}

Durch eine ausschließliche Bewertung am Ende einer Lektion werden viele Lernpotenzen, die Bewertungen und Rückmeldungen in sich bergen, verschenkt. Deshalb sollten die Kinder während des gesamten Lernprozesses

- Fehler als Lernquelle nutzen lernen,

$\circ$ Lernziele und Lernkriterien kennen und darauf immer wieder Bezug nehmen,

○ regelmäßige Rückmeldungen (vom Lehrer, von Mitschülern, durch Selbst-Überprüfung) über ihren Lernstand erhalten,

○ häufige Gelegenheiten zum kritischen Nachdenken bekommen,

○ Gelegenheiten haben, über ihre Gefühle beim Lernen zu sprechen,

○ Möglichkeiten haben, ihre Arbeit zu qualifizieren.

Vom Beginn bis zum Ende einer Lektion wird den Kindern die Möglichkeit eingeräumt, ihr aktuelles Verstehen zum Ausdruck zu bringen. Die Lernprozesse sind so gestaltet, dass die Kinder von eigenen Erfahrungen ausgehend den Übergang zu ähnlichen Ereignissen finden können. Immer wieder werden sie angeregt, Teilereignisse mit eigenen Worten zu erklären, zu spekulieren, was geschehen wäre bzw. würde, wenn die Bedingungen andere gewesen wären. Die Lernkontrollen sind weniger in Form von Tests (die natürlich nicht auszuschließen sind), sondern in Form von individuellen und kreativen Präsentationen - wie der Gestaltung eines Posters, einer Collage, einer Ausstellung, eines Vortrages, dem Abfassen einer essayähnlichen Niederschrift, der Dokumentation eines Projektes, dem Anlegen eines Portfolios usw. durchzuführen. Eine das Lernen begleitende Leistungsdarstellung muss die Kinder in die Lage versetzen, tatsächlich zu erkennen, was sie gelernt haben und wie sie ihr Wissen kreativ beim Lösen von Problemen und zur Herausbildung eines neuen Verständnisses nutzen können. Um zu prüfen, ob die Schüler das Problem, den Inhalt verstanden haben, sollten sie ihre Gedanken immer auf mindestens zwei unterschiedlichen Wegen zum Ausdruck bringen. Kinder brauchen ein passendes Maß an persönlicher Kontrolle und Unabhängigkeit, das sich mit ihrer wachsenden Kompetenz immer mehr vom Lehrer 
auf sie selbst verschiebt. Eine wichtige Rolle spielen dabei die aus der Schülerperspektive formulierten Lernziele und Lernkriterien.

\section{Reflexionen über das Lernen}

Alle gegenstandsbezogenen kognitiven Lernprozesse sind mit metakognitivem Lernen, dem Lernen des Lernens, zu verknüpfen. Entsprechende Reflexionen unterstützen die reziproken Beziehungen zwischen Lernprozess und Lernergebnissen. Dazu können die Kinder z. B. Lerntagebücher führen oder ein Portfolio erstellen. Anwendungsbezogene Aufgaben, bei denen ein Problem zu lösen ist, das Kinder in ihrer Lebenswelt nachvollziehen können, geben Aufschluss über die Transferfähigkeit des angeeigneten Wissens. Gerade die Ausbildung eines transferfähigen Wissens ist ein wesentlicher Baustein metakognitiver Kompetenzen (Revákné-Markóczi 2007). Jede Lerneinheit ist mit einer ausführlichen Reflexionsphase abzuschließen. Die Kinder werden aufgefordert, ihr persönliches Erreichen der Lernziele unter Zuhilfenahme der Lernkriterien einzuschätzen:

- Was habe ich gelernt?

- Welche Fragen habe ich noch dazu?

$\circ$ Welche Aufgaben waren für mich schwierig?

- Hatte ich die Möglichkeit etwas zu gestalten?

Neben diesen Fragen, die sich vor allem auf den Lerngegenstand beziehen, werden auch Fragen, die sich mit den emotionalen Befindlichkeiten der Kinder während des Lernprozesses befassen, thematisiert. Der Lehrer inspiriert den Gedankenaustausch z.B. durch folgende Impulse:

- Wie hast du dich gefühlt, als du:

- eine Aufgabe erfolgreich gelöst hattest?

- Schwierigkeiten bei einer Aufgabe hattest?

- um Hilfe gebeten hast?

- Hilfe bekommen hast?

○ Fühltest du dich in der Gruppe anerkannt und wurde dein Beitrag geschätzt?

○ Welche Atmosphäre in der Klasse unterstützt dich beim Lernen?

\section{Stärkung des Selbstwertgefühls eines jeden Kindes}

Schulische Lernprozesse werden von subjektiven Faktoren maßgeblich beeinflusst. Dazu gehört auch das Selbstwertgefühl, das Kinder für ihre Person entwickeln. Sie möchten sich kompetent fühlen, d.h. sie möchten wissen und verstehen und in der Lage sein, die gestellten Aufgaben gut zu erledigen. Die pädagogisch-didaktische Reflexion subjektiver Lernfaktoren ist unter dem Stichwort ,personalised learning“ zusammengefasst (Hodson 1998; Charles 2000; Dean 2006). Alle Maßnahmen sind deshalb darauf zu richten, das 
Selbstwertgefühl eines jeden Kindes zu stärken. Darunter ist ein psychologisches Konstrukt zu verstehen, das die gefühlsmäßig positiv oder negativ orientierte Selbstbewertung eines Menschen ausdrückt (Damon 1989). Es beruht auf intra- oder interindividuell vergleichenden und emotional erlebten situativen und bereichsspezifischen Erfahrungen, die sich über Zeit und Bereiche generalisieren und zu einem grundlegenden Gefühl für die Person werden (Haußer 1995). Das Streben nach einer positiven Selbstwertung gehört zu den menschlichen Grundbedürfnissen und wirkt verhaltensdeterminierend. Beim Selbstwertgefühl sind - in Abhängigkeit der Bewertungsrichtung - drei Kategorien zu unterscheiden: Selbstvertrauen, Vertrauen und Selbstbewusstsein (Schneider 2003, 42ff.).

Das Selbstvertrauen gibt Auskunft über das subjektive Empfinden der eigenen Fähigkeiten und Eigenschaften. Es entwickelt sich in einem Spannungsfeld, das durch die Pole Zuversicht und Unsicherheit gekennzeichnet ist. Die gesamte Atmosphäre im Klassenraum und die Art und Weise, wie Leistungen gewürdigt werden, beeinflussen das Selbstvertrauen der Kinder. Deshalb sollte im Verlauf eines Lernprozesses immer der individuelle Lernfortschritt Maßstab der Bewertung sein. Die Leistungen der Schüler sind nicht untereinander zu vergleichen. Fehler sind grundsätzlich nicht als Mangel, sondern als Lernpotenz zu verstehen.

Vertrauen bringt das Sicherheitsbedürfnis eines Menschen zum Ausdruck. Eine Person richtet ihre Aufmerksamkeit auf eine andere Person, von der sie die Befriedigung dieses Bedürfnisses erwartet. Das ist in der Schule in hohem Maße die Lehrerin. Eine wichtige Voraussetzung für die Herausbildung von vertrauensvollen Beziehungen ist die gegenseitige Achtung der körperlichen und seelischen Integrität der Persönlichkeit. Im Vertrauen wird ein Bedürfnis nach Bindung, Anlehnung und Orientierung sichtbar.

Im Gegensatz dazu betont die Kategorie Selbstbewusstsein die gefühlsmäßige Einschätzung des eigenen Handlungspotentials und darauf basierende Kompetenzzuschreibungen und Handlungsbereitschaften. Die Art und Weise, wie eigenes Verhalten und eigene Handlungen in Bezug auf andere reflektiert werden, führen zu einem emotionalen Grundgefühl, das dem eigenen Ich in Distanzierung zu anderen einen bestimmten Wert zumisst und zu einer selbst bestimmten Handlungsbereitschaft führt. Das kann sich z.B. im Durchsetzungsanspruch, Behauptungsstreben, in Wertzumessungen und Abgrenzungsbemühungen äußern. Die konsequente Einbeziehung der Kinder in die Gestaltung ihrer Lernprozesse stärkt ihr Selbstwertgefühl, und ein positives Selbstwertgefühl ist ein nicht zu unterschätzender Lernfaktor. 


\subsubsection{Muster für die Planung einer Lerneinheit nach dem Rostocker Modell}

\begin{tabular}{|c|c|c|}
\hline \multicolumn{3}{|c|}{ Lernmodul „Wir leben in Europa“ für die Klassen 3/4 } \\
\hline \multicolumn{3}{|c|}{ Leitidee: Je besser wir uns kennen, desto besser können wir uns verstehen. } \\
\hline 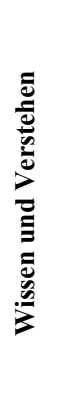 & $\begin{array}{l}\text { 1. Was ist die EU und welche Länder gehören } \\
\text { dazu? (Die Kinder wissen, was EU bedeutet und } \\
\text { können die Mitgliedsstaaten nennen und auf der } \\
\text { Karte zeigen.) } \\
\text { 2. Was wissen wir über die Mitgliedsländer der } \\
\text { EU? (Die Kinder kennen die Hauptstädte, die } \\
\text { Staatsflaggen und geografische Besonderheiten } \\
\text { dieser Länder.) } \\
\text { 3. Wie leben Kinder in EU-Ländern? (Die Kinder } \\
\text { erkennen, dass die Sitten und Bräuche von Land } \\
\text { zu Land verschieden sind.) }\end{array}$ & $\begin{array}{l}\text { Hauptbegriffe: } \\
\text { Europa } \\
\text { EU } \\
\text { Land } \\
\text { Hauptstadt } \\
\text { Gemeinsamkeiten } \\
\text { Unterschiede } \\
\text { Gegenseitige Achtung }\end{array}$ \\
\hline 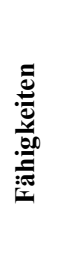 & $\begin{array}{l}\text { Die Kindern entwickeln die Fähigkeit, } \\
\text { - Medien zielgerichtet als Informationsquellen zu } \\
\text { nutzen } \\
\text { - Fakten und Aussagen miteinander zu verglei- } \\
\text { chen und zu bewerten } \\
\text { - Informationen zu präsentieren } \\
\text { - ein Portfolio zu erstellen. }\end{array}$ & \multirow[t]{2}{*}{$\begin{array}{l}\text { Portfolio } \\
\text { Tabelle } \\
\text { Poster }\end{array}$} \\
\hline 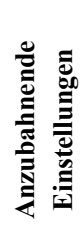 & $\begin{array}{l}\text { Die Kinder entwickeln das Bedürfnis, } \\
\text { - sich über Europa zu informieren. } \\
\text { - mehr darüber zu erfahren, wie Kinder in ande- } \\
\text { ren europäischen Ländern leben. } \\
\text { - Kinder aus anderen europäischen Ländern } \\
\text { kennen lernen zu wollen. }\end{array}$ & \\
\hline $\begin{array}{l}\text { Lern- } \\
\text { phase }\end{array}$ & Lerntätigkeiten/Inhalte & $\begin{array}{l}\text { Lernfördernde Rückmel- } \\
\text { dungen }\end{array}$ \\
\hline \multirow{2}{*}{ 总 } & $\begin{array}{l}\text { a. Die Lehrerin führt in die Arbeit eines Portfo- } \\
\text { lios ein (LI). }\end{array}$ & $\begin{array}{l}\text { Jedes Kind sammelt } \\
\text { seine Arbeitsergebnisse } \\
\text { in einem Portfolio und } \\
\text { reflektiert diese. }\end{array}$ \\
\hline & $\begin{array}{l}\text { b. Was bedeutet Europa im Alltag? } \\
\text { Es werden Arbeitsgruppen gebildet. Jede Gruppe } \\
\text { konzentriert sich auf ein europäisches Land (P). } \\
\text { Die Gruppen besuchen einen Supermarkt. Die } \\
\text { Kinder ermitteln, welche Lebensmittel aus ihrem }\end{array}$ & $\begin{array}{l}\text { Die Lehrerin bereitet für } \\
\text { jede Gruppe eine Tabelle } \\
\text { vor. } \\
\text { HA: Ermittle zu Hause } \\
\text { drei weitere Lebensmit- }\end{array}$ \\
\hline
\end{tabular}




\begin{tabular}{|c|c|c|}
\hline & $\begin{array}{l}\text { gewählten europäischen Land stammen (PA). } \\
\text { Die Lehrerin entwickelt mit den Kindern eine } \\
\text { Tabelle, in der die Ergebnisse eingetragen wer- } \\
\text { den (EA). } \rightarrow \text { Portfolio }\end{array}$ & $\begin{array}{l}\text { tel, die aus einem euro- } \\
\text { päischen Land stammen. }\end{array}$ \\
\hline & $\begin{array}{l}\text { c. Die HA wird verglichen }(\mathrm{P}) \text {. } \\
\text { Die Kinder ergänzen ihre Eintragungen in der } \\
\text { Tabelle (GA). } \rightarrow \text { Portfolio }\end{array}$ & $\begin{array}{l}\text { Die Lehrerin achtet auf } \\
\text { die richtige Rechtschrei- } \\
\text { bung. }\end{array}$ \\
\hline & $\begin{array}{l}\text { d. Lehrerin und Kinder diskutieren die Lernbe- } \\
\text { deutung, Lernziele und Lernkriterien (KG) } \rightarrow \\
\text { Portfolio }\end{array}$ & $\begin{array}{l}\text { Die Lehrerin achtet auf } \\
\text { die Einhaltung der } \\
\text { Gesprächsregeln, Lern- } \\
\text { bedeutung, Lernziele } \\
\text { und Lernkriterien wer- } \\
\text { den besprochen }\end{array}$ \\
\hline \multirow{5}{*}{ 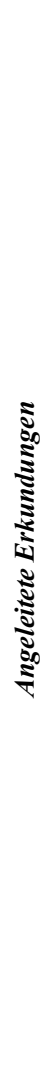 } & $\begin{array}{l}\text { e. Länder und Hauptstädte } \\
\text { Die Kinder ermitteln unter Nutzung von Nach- } \\
\text { schlagwerke und Internet, welche Länder zur } \\
\text { Europäischen Union gehören und deren Haupt- } \\
\text { stadt (PA). } \rightarrow \text { Portfolio } \\
\text { Die Lehrerin erklärt die Begriffe „Europa“ und } \\
\text { „Europäische Union“(LI). }\end{array}$ & $\begin{array}{l}\text { Die Lehrerin stellt } \\
\text { Nachschlagwerke zur } \\
\text { Verfügung (z.B. Meyers } \\
\text { Länderlexikon für Kin- } \\
\text { der) und hilft ggf. bei der } \\
\text { Internetsuche. } \\
\text { HA: Welche Kontinente } \\
\text { gibt es auf der Erde? } \rightarrow \\
\text { Portfolio }\end{array}$ \\
\hline & $\begin{array}{l}\text { f. Vergleich der HA }(\mathrm{P}) \text {. } \\
\text { Die Kinder benutzen Nachschlagwerke und } \\
\text { Internet, um die Landesfahnen der einzelnen EU- } \\
\text { Mitgliedsstaaten zu identifizieren (PA). }\end{array}$ & $\begin{array}{l}\text { Die Lehrerin unterstützt } \\
\text { die Medienarbeit (Kin- } \\
\text { dersuchmaschinen: z.B. } \\
\text { www.blinde-kuh.de). } \rightarrow \\
\text { Portfolio }\end{array}$ \\
\hline & $\begin{array}{l}\text { g. Gebirge }- \text { Berge }- \text { Flüsse } \\
\text { Auf die gleiche Weise bestimmen die Kinder z.B. } \\
\text { die fünf längsten Flüsse, die fünf größten Gebir- } \\
\text { ge, die fünf höchsten Berge in Europa (PA). } \rightarrow \\
\text { Portfolio }\end{array}$ & $\begin{array}{l}\text { z.B. Buch aus der Reihe } \\
\text { WAS-IST-Was, Bd. 113: } \\
\text { Europa } \\
\text { Spiel: Stadt-Land-Fluss }\end{array}$ \\
\hline & $\begin{array}{l}\text { h. Leben in Europa } \\
\text { Die Kinder tauschen sich über ihre Erfahrungen } \\
\text { mit anderen Ländern aus (KG). }\end{array}$ & $\begin{array}{l}\text { Die Lehrerin erzählt von } \\
\text { ihren eigenen Erfahrun- } \\
\text { gen, Einsatz von Kinder- } \\
\text { büchern }\end{array}$ \\
\hline & $\begin{array}{l}\text { i. Leben in Europa } \\
\text { Die Kinder erkunden durch Befragungen und } \\
\text { Medienrecherchen, wie Kinder in anderen euro- } \\
\text { päischen Ländern leben: Schule, Spiele, Lieb- } \\
\text { lingsessen usw. (GA). }\end{array}$ & $\begin{array}{l}\text { Die Lehrerin unterstützt } \\
\text { die Medienarbeit (z.B. } \\
\text { www.google.de). }\end{array}$ \\
\hline
\end{tabular}




\begin{tabular}{|c|c|c|}
\hline & $\begin{array}{l}\text { j. Die Lehrerin erarbeitet mit den Kindern eine } \\
\text { Tabelle, um die Ergebnisse miteinander zu } \\
\text { vergleichen }(\mathrm{P}) . \rightarrow \text { Portfolio }\end{array}$ & $\begin{array}{l}\text { Sie achtet auf die richti- } \\
\text { ge Rechtschreibung. }\end{array}$ \\
\hline & $\begin{array}{l}\text { k. Die Lehrerin oder Mitschüler erklären Spiele, } \\
\text { die Kinder in anderen Ländern spielen (LI; SV). } \\
\text { Die Kinder beschäftigen sich mit Märchen aus } \\
\text { verschiedenen Ländern. } \rightarrow \text { Portfolio }\end{array}$ & $\begin{array}{l}\text { Die Kinder probieren } \\
\text { diese Spiele aus. Welche } \\
\text { Märchen ähneln sich? } \\
\text { (P) } \\
\text { Vorstellung des Lieb- } \\
\text { lingsmärchens (SV). }\end{array}$ \\
\hline & $\begin{array}{l}\text { 1. Gemeinsam werden typische Landesspeisen } \\
\text { hergestellt und ausprobiert. } \\
\text { Jedes Kind wählt ein Rezept aus und übernimmt } \\
\text { es in sein } \rightarrow \text { Portfolio (EA). }\end{array}$ & $\begin{array}{l}\text { Die Lehrerin bittet Eltern } \\
\text { um Unterstützung. }\end{array}$ \\
\hline & $\begin{array}{l}\text { m. Die Kinder befragen ihre Eltern und Großel- } \\
\text { tern, wie diese als Kinder Europa erlebt haben } \\
\text { (EA). Sie vergleichen deren Erlebnisse mit ihren } \\
\text { eigenen Erfahrungen. } \rightarrow \text { Portfolio. }\end{array}$ & z.B. Fotoalben \\
\hline \multirow{2}{*}{ 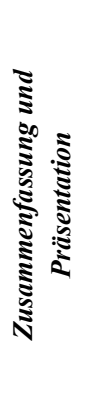 } & $\begin{array}{l}\mathrm{n} \text {. Jedes Kind wählt ein EU-Land, mit dem es } \\
\text { sich besonders beschäftigen möchte. Es nutzt } \\
\text { seine Aufzeichnungen aus dem Portfolio und } \\
\text { sucht nach weiteren Informationen (EA). Die } \\
\text { Lehrerin gibt Schwerpunkte zur Orientierung vor } \\
\text { (z.B. Essen, Spiele, Märchen, Feste feiern, ...) }\end{array}$ & $\begin{array}{l}\text { Die Lehrerin ermuntert } \\
\text { die Kinder, nach etwas } \\
\text { Besonderem Ausschau } \\
\text { zu halten, um die ande- } \\
\text { ren ,zu überraschen“. } \\
\text { Sie hält entsprechende } \\
\text { Kinderbücher bereit. }\end{array}$ \\
\hline & $\begin{array}{l}\text { o. Jedes Kind stellt sein gewähltes Land auf } \\
\text { einem Poster (EA) den anderen Kindern vor (P; } \\
\text { SV). }\end{array}$ & $\begin{array}{l}\text { Die Lehrerin gibt indivi- } \\
\text { duelle Hilfe. Die Kinder } \\
\text { geben sich untereinander } \\
\text { ein Feedback }\end{array}$ \\
\hline$\frac{\approx}{\sqrt[5]{5}}$ & $\begin{array}{l}\text { p. Die Kinder diskutieren z.B. folgende Fragen: } \\
\text { - Was habe ich gelernt? } \\
\text { - Was/wer hat mir beim Lernen geholfen? } \\
\text { - Was habe ich noch nicht verstanden? } \\
\text { - Was möchte ich noch wissen? } \\
\text { - ...(KG) }\end{array}$ & $\begin{array}{l}\text { Die Lehrerin verweist } \\
\text { auf die Lernziele und } \\
\text { Lernkriterien. Sie stellt } \\
\text { Fragen zum Lernprozess } \\
\text { und nutzt die Einschät- } \\
\text { zung der Kinder für ihre } \\
\text { Lernanalyse. }\end{array}$ \\
\hline
\end{tabular}

* Legende zu den Abkürzungen: EA = Einzelarbeit; PA = Partnerarbeit; GA = Gruppenarbeit; $\mathrm{KG}=$ Kreisgespräch; LI = Lehrerinstruktion; SV = Schülervortrag; HA = Hausaufgabe 


\section{Literatur}

Berger, P. L.\& Luckmann, T. (1991): Die gesellschaftliche Konstruktion der Wirklichkeit. Frankfurt a. M.: Fischer Taschenbuch Verlag

Blythe, T. (1998): The Teaching For Understanding Guide. San Francisco: Jossey-Bass Publisher

Bruner, J. (1973): Der Prozeß der Erziehung. Berlin: Berlin Verlag

Carey, S. (1985): Conceptual change in childhood. Cambridge, MA: The MIT Press

Charles, C. M. (2000): The Synergetic Classroom. Joyful Teaching and Gentle Disscipline. New York: Longman

Clarke, Sh. (2001): Unkocking Formative Assessment. Practical strategies for enhancing pupils' learning in the primary classroom. London: Hodder \& Stougthon

Csikszentmihalyi, M. (1985): Das flow-Erlebnis. Jenseits von Angst und Langeweile: im Tun aufgehen. Stuttgart: Klett-Cotta

Damon, W. (1989): Die soziale Entwicklung des Kindes. Stuttgart: Klett-Cotta

Dean, J. (2006): Meeting the learning needs of all children. Personalised learning in the primary school. London, New York: Routledge

Duit, R. (1997): Alltagsvorstellungen und Konzeptwechsel im naturwissenschaftlichen Unterricht - Forschungsstand und Perspektiven für den Sachunterricht in der Primarstufe. In: Köhnlein, W. u. a. (Hrsg.): Kinder auf dem Wege zum Verstehen der Welt. Bad Heilbrunn: Klinkhardt, 233-246

Gardner, H. (1994): Der ungeschulte Kopf. Wie Kinder denken. Stuttgart: Klett-Cotta

Gopnik, A. u. a. (2000): Forschergeist in Windeln. Wie Ihr Kind die Welt begreift. München: Ariston

Haußer, K. (1995): Identitätspsychologie. Berlin: Springer

Heran-Dörr, E. (2006): Orientierung an Schülervorstellungen - Wie verstehen Lehrkräfte diesen Appell an ihre didaktische und methodische Kompetenz? In: Cech, D.; Fischer, H.-J.; Knörzer, M. \& Schrenk, M. (Hrsg.): Bildungswert des Sachunterrichts. Bad Heilbrunn: Klinkhardt, 159-176

Hodson, D. (1998): Teaching and Learning Science. Towards a personalized approach. Buckingham, Philadelphia: Open University Press

Hofstein, A. \& Kempa, R. F. (1985): Motivating strategies in science education: attempt at an analysis, European Journal of Science Education, 7, 221-229

Klafki, W. (1957): Das pädagogische Problem des Elementaren und die Theorie der kategorialen Bildung. Weinheim, Basel: Beltz

Klafki, W. (1985): Neue Studien zur Bildungstheorie und Didaktik. Beiträge zur kritischkonstruktiven Didaktik. Weinheim, Basel: Beltz

Klafki, W. (1992): „Schlüsselprobleme“ als thematische Dimension eines zukunftsorientierten Konzepts von Allgemeinbildung - Zwölf Thesen. In: Münzinger, W. \& Klafki, W. (Hrsg.): Schlüsselprobleme im Unterricht. Die deutsche Schule, 3. Beiheft, 9-14

Kempa, R. F. \& Diaz, M. M. (1990): Motivational traits and preferences for different instructional modes in science. Part 1 and 2, International Journal of Science Education, 17, 743-754

Krnel, D. (2005): The Development of the Concept of Matter': A Cross study of how children describe materials. International Journal of Science Education, 3, 367-383

Novak, J. D. (1998): Learning, Creating and Using Knowledge. Concept Maps as Facilitative Tools in Schools and Corporations. London: Lawrence Erlbaum

Otto, B. (1965): Ratschläge für den häuslichen Unterricht. Heidelberg: Quelle \& Meyer

Poddjakow, N. N. (1981): Die Denkentwicklung beim Vorschulkind. Berlin: Volk und Wissen

Revákne-Markóczi, I.; Kosztin-Tóth; B.; Tóth, Z.; Dobó-Tarai, E.; Schneider, I. K. \& Oberländer, F. (2008). Effects of the Rostock Model on Metacognitive Development of Pupils. La 
influenca del modelo Rostock sobre el desarrollo metacognitivo de los alumnus. Journal of Science Education, 2, 94-99

Schneider, I. K. (2000): Philosophieren mit Kindern. Potenzen auch für eine politische Bildung? In Grundschule, 7-8, 68-70

Schneider, I. K. (2003): So sehe ich die Sache! Kinder verstehen - Kinder erziehen. Baltmannsweiler: Schneider

Schneider, I. K. (2007): Politische Bildung in der Grundschule. Sachinformationen, didaktische und methodische Überlegungen, Unterrichtsideen und Arbeitsmaterialien für die 1. bis 4 . Klasse. Baltmannsweiler: Schneider

Schneider, I. K. \& Oberländer, F. (2008): Naturwissenschaftliches Lernen in der Grundschule. Was Kinder können - was Kinder brauchen. Lerneinheiten zum Thema „Wasser“ für die Klassen 1 bis 4 mit ausgearbeiteten Experimenten, Arbeitsblättern, Protokollvorlagen und Lehrerinformationen. Baltmannsweiler: Schneider

Schneider, I. K.; Hruby, A. \& Pentzien, S. (2007): Naturwissenschaftliches Lernen in der Grundschule. Lerntheoretische didaktische und methodische Überlegungen, Unterrichtsideen, Arbeitsmaterialien und Experimentieranleitungen für die 1. und 2. Klasse. Donauwörth: Auer

Schneider, I. K.; Oberländer, F.; Tóth, Z.; Dobó-Tarai, E.\& Revák-Markóczi, I. (2008a): Scientific Learning in Primary School Education - A Model Study on Children's Concepts of Physical Material. Practice and Theory in Systems of Education, 2, 1-23

Schneider, I. K.; Oberländer, F.; Tóth, Z.; Dobó-Tarai,E. \& Revák-Markóczi, I. (2008b): Naturwissenschaftliches Lernen - eine exemplarische Studie zur Entwicklung von Stoffkonzepten. In Problemy Wczesnej Edukacji. Gdansk: Universität Gdansk, 8, 112-120

Schneider, I. K. \& Oberländer, F. (2009): Naturwissenschaftliches Lernen im Anfangsunterricht - eine exemplarische Studie zu Stoffkonzepten. In: Lauterbach, R.; Giest, H. \& MarquardtMau, B. (Hrsg.): Lernen und kindliche Entwicklung. Elementarbildung und Sachunterricht Bad Heilbrunn: Klinkhardt, 205-211

Singer, W. (2003): Ein neues Menschenbild? Gespräche über Hirnforschung. Frankfurt a. M.: Suhrkamp

Spitzer, M. (2004): Selbstbestimmen. Gehirnforschung und die Frage: Was sollen wir tun? Heidelberg, Berlin: Spektrum Akad. Verlag

Tóth, Z., Dobó-Tarai, E., Revák-Markóczi, I., Schneider, I. K. \& Oberländer, F. (2007): 1st graders prior knowledge about water: knowledge space theory applied to interview data. Journal of Science Education, 2, 116-119

Tóth, Z., Dobó-Tarai, E., Revák-Markóczi, I., Schneider, I. K., Oberländer, F. (2008): Effects of instruction on 1st graders' thinking patterns regarding the description of water with every day and scientific concepts. Practice and Theory in Systems of Education, 1, 45-54

West, L. H. \& Pine, A. L. (1983): How 'rational' is rationality? Science Education, 67, 37-39

Vygotskij, L. S. (2002): Denken und Sprechen. Weinheim: Beltz 